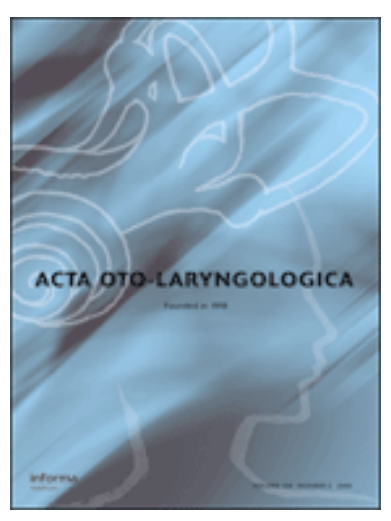

\title{
BONE CONDUCTIVE IMPLANTATION IN ASYMMETRIC HEARING LOSS (AHL)
}

\begin{tabular}{|r|l|}
\hline Journal: & Acta Oto-Laryngologica \\
\hline Manuscript ID & Draft \\
\hline Manuscript Type: & Regular \\
\hline Classification Scheme: & audiology \\
\hline Keywords: & $\begin{array}{l}\text { Bone conductive implants, Asymmetric Hearing loss, Single sided } \\
\text { deafness }\end{array}$ \\
\hline
\end{tabular}

\section{SCHOLARONE ${ }^{m}$ Manuscripts}


BONE CONDUCTIVE IMPLANTATION IN ASYMMETRIC HEARING LOSS (AHL)

Keywords: Bone Conductive Device; single sided deafness; Asymmetric hearing loss 


\begin{abstract}
Background: Bone conductive implants (BCI) represent one possible solution for rehabilitation of single sided deafness (SSD)

Aims: The aim of the present study was to verify the efficacy of bone conduction implantation in subjects with unilateral severe-to-profound hearing loss and contralaterally-impaired hearing loss, i.e. asymmetric hearing loss (AHL), and to compare it with known BCI indications for SSD.

Material and Methods: Twenty-one subjects received BCI for either SSD or AHL. All of the subjects underwent a battery of audiological and subjective tests, Data were collected and statistically evaluated within and between the SSD group and the AHL group.

Results: A PTA threshold gain was observed in AHL patients along with improved values in speech audiometry in quiet and noise. The two visual-analogue-scale evaluations (QoL and QoS) and the GBI showed significantly better scores in AHL patients compared to SSD patients.
\end{abstract}

Conclusions: BCI did not provide improvement for auditory or speech recognition in SSD subjects, but it did it, however, for the AHL subjects. From these findings, it is possible to predict a positive role of BCI for some audiological aspects of AHL subjects that are generally not present in SSD cases 


\section{INTRODUCTION}

Single-Sided Deafness (SSD) is defined as a unilateral, severe (pure-tone average $>70 \mathrm{~dB}$ ) sensorineural loss of hearing with a normally functioning contralateral ear and is estimated to affect between $7.9 \%$ and $13.3 \%$ of the world's hearing-impaired population [1]. When the contralateral ear also has a functional impairment of an at least $15 \mathrm{~dB}$ threshold difference from the worse hearing ear, the term Asymmetric Hearing Loss (AHL) is used [2-4].

The complete loss of auditory function in one ear, called anacusis, causes an individual to miss important acoustic cues that are used by those with binaural hearing: binaural summation (approximately $10 \mathrm{~dB}$ ), the head-shadow effect that, via differences in intensity and time perception, allows one to localise a sound source, and the squelch effect, which allows one to separate the target message from background noise [5].

Different rehabilitation tools are available today to overcome all or part the disadvantages of loss of binaural hearing; they include conventional contralateral route of signal (CROS) hearing aids, bone conductive implants (BCIs) [6, 7] and cochlear implants (CIs) [8]. Most authors agree that, for SSD rehabilitation, a BCI represents a practicable option, especially for the expansion of the sound field, although it is unable to reproduce true binaural hearing [9-13]. More specifically, BCI attenuates the head-shadow effect via bone transmission without affecting the basal monoaural advantages and provides an artificial shadow effect that could be advantageous for directional hearing [11]. The advantage of rehabilitating a deaf ear via $\mathrm{BCI}$ is related to the possibility of combining, at the cortical level, information coming from the same affected cochlea through the contralateral ear, although with a temporal delay that may only mimic a true binaural hearing.

Previous audiological studies have investigated the advantages of BCIs for unilateral deafness and have revealed that, in SSD subjects, they do not provide a remarkable hearing gain through 
stimulation of the contralateral cochlea, as shown in speech audiometry by the optimal hearing (ceiling effect) provided by the normal contralateral ear. By contrast, in AHL cases where the ceiling effect is absent, an improved speech perception has been reported after BCI implantation $[7,13]$. However, communication under disadvantageous conditions, such as in noisy environments, only improves when the sound stimulates the implanted-ear side and noise is on the non-implanted side, which is not always the case in daily situations [13].

The present study was designed to assess the efficacy of BCI in subjects with profound unilateral hearing loss and mild-to-moderate hearing loss in the contralateral ear, both of which are considered to be features that define an AHL. In particular, both BCI-aided SSD and AHL subjects were compared to each other and with the unaided condition, by assessing verbal perception in quiet and in noise and by investigating the subjectively perceived quality of sound. 


\section{MATERIALS AND METHODS}

This retrospective study included 21 subjects (9 females, 12 males; mean age 55.6) who underwent BCI implantation at a tertiary university hospital between 2010 and 2018. A preliminary pure-tone audiometry allowed us to distinguish the subjects into either SSD or AHL group (Table I). According to the routine audiological approach applied to all BCI candidates, all subjects underwent an assisted audiometric evaluation before the operation in which a headband-held BCI sound processor (SP) was chosen according to the individual's hearing loss to predict the post-operative outcome [14]. For this test the following SPs were used: Divino (5 subjects), Intenso (1 subject), BP100 (6 subjects), BAHA4 (8 subjects), and BAHA5 (1 subject). Divino and Intenso SPs were fitted manually; the SSD-software provided by the manufacturer (Cochlear, Melbourne, Australia) was used for the others. The worse ear was always selected for implantation, and activation and fitting of the implant were performed approximately 45 days after surgery, when the subjects received the following battery of functional tests under the unaided and BCI-aided situations:

- Free-field pure-tone audiometry to assess the Pure Tone Average (PTA) from 0.25 to 4 kHz.

- Free-field speech audiometry in quiet to assess:

o the maximum Word Recognition Score (WRS) in percent of intelligibility and the relative $\mathrm{dB} H \mathrm{HL}$,

- WRS at $65 \mathrm{~dB}$ HL,

o and Speech Reception Threshold (SRT, dB HL for 50\% of intelligibility).

The speech material comprised sequences of 10 bi- and three-syllabic words balanced in consonant and vowel content. 
- Free-field speech audiometry in noise to assess the maximal percentage of intelligibility at a signal-to-noise ratio $+10(\mathrm{~S} / \mathrm{R}+10)$. During this test, loudspeakers delivered noise to the non-implanted ear and the signal to the implanted ear.

In both SSD and AHL subjects, the values under the unaided and BCI-aided situations were compared, taking into consideration:

- absolute PTA values in both situations;

- hearing gain in the BCI-aided versus the unaided condition;

- and gain for the selected frequencies in the two groups.

For speech audiometry in quiet, the absolute WRS values and percent of intelligibility, WRS at $65 \mathrm{~dB}$ HL (percent of intelligibility at $65 \mathrm{~dB}$ ), and the SRT (50\% of intelligibility) were assessed. Moreover, the eventual improvement in the percent of intelligibility and loudness for the three parameters were compared in the unaided and BCI-aided conditions. For speech audiometry in noise, the evaluation included the shift in the percent of intelligibility between the unaided and the BCI-aided situation at the level of stimulation (in $\mathrm{dB}$ HL) at the level used for WRS maximum of speech audiometry in quiet. Finally, an evaluation of the gain and improvement differences in speech performance in quiet and noise between the two study groups was performed.

\section{Subjective Evaluations}

Three months after BCI activation, all subjects underwent the following tests:

- Visual Analog Scale (VAS). Two scales from 0 to 10 were used, with one measuring the subjective perception of the perceived sound (open or clear), and the other measuring quality of life (QoL). The score was considered positive if greater than 6 and excellent if greater than 8. 
- General Glasgow Benefit Inventory (GBI) (standardised Italian version) is a questionnaire used for the evaluation of life quality after a surgical operation that consists of 18 items investigating changes in health status after surgery (12 questions on general health, 3 on physical health and 3 on social health). For each question, five answers are available, with a score from 1 to 5 , for a possible final score between -100 and 100, calculated by dividing the total score by the number of questions, subtracting 3, and multiplying by 50 [15].

- Entific Medical System QoL is a questionnaire proposed by the former BCI-owner company (Entific, Gothenburg, Sweden) to evaluate the general satisfaction of BCI users [16]. It consists of two sections, the Q26A and the Q26B; each section is composed of 25 questions. Q26A investigates improvement in different listening situations; Q26B investigates patient satisfaction after an adaptation period of approximately 3 months. For each section, items are scored from 1 to 5, with a minimum total score of 25 and a maximum of 125 .

\section{Statistical Analysis of Subjective Tests}

Statistical analysis examined the significance of the comparison of unaided versus BCI-aided situations in each group for every performed test.

To compare the unaided and BCI-aided situations within each group, the binomial test or multiple group test was used and applied to all parameters: single frequency PTA, maximum percent of WRS, maximum dB HL WRS, SRT, WRS at $65 \mathrm{~dB}$ HL, and percent of intelligibility with $\mathrm{S} / \mathrm{N}$ ratio $+10 \mathrm{~dB}$ HL.

To compare the two groups, the Mann-Whitney U test was used for every parameter to demonstrate:

- eventual homogeneity of the two groups based on the hearing level of the contralateral ear; 
- significance of the comparison between the two groups, in unaided and BCI-aided situations, for PTA gain at each frequency and speech audiometry scores in quiet and in noise. The results were considered positive for values less than $0.05(\mathrm{p}<0.05)$.

\begin{abstract}
All the subjects under study received and signed the relative informed consent. The study obtained appropriate institutional review board approval from the university hospital and followed the recommendations of the Declaration of Helsinki for biomedical studies involving human subjects [17].
\end{abstract}




\section{RESULTS}

The mean audiometric thresholds of the contralateral (better hearing) ear in SSD and AHL subjects is shown in Figure 1. For SSD patients, the mean air-conduction threshold was 17.7 $\mathrm{dB} H \mathrm{HL}$ and the mean bone-conduction threshold was $13.3 \mathrm{~dB}$ HL. For AHL patients, the values were 40.4 and $28.5 \mathrm{~dB}$ HL, respectively.

The two groups were non-homogenous in regard to the mean auditory threshold of the nonimplanted ear, with a statistically significant difference $(p<0.05)$, as confirmation of the clearcut distinction between the two groups.

\section{Free-Field Pure-Tone Audiometry}

The individual and mean PTA values $(0.25-4 \mathrm{kHz})$ and the relative gain in the BCI-aided situation are shown in Table II and Figure 2. In SSD patients, the mean PTA shifted from 37.5 dB HL (unaided) to $34.9 \mathrm{~dB}$ HL (BCI-aided). In AHL patients, the mean PTA shifted from $60.4 \mathrm{~dB}$ HL (unaided) to $55.3 \mathrm{~dB}$ HL (BCI-aided). The mean gain was $2.5 \mathrm{~dB}$ HL in SSD patients and 5.1 dB HL in AHL patients, with a 2.6 dB HL difference between the two groups (AHL $p<0.000$; SSD $p<0.005)$. The mean gain at each frequency indicated a greater gain in the SSD group between 0.5 and $2 \mathrm{kHz}$ and a greater gain in the AHL group at 1 and, particularly, at $4 \mathrm{kHz}$ (Figure 3). The single frequency gain was not significant $(\mathrm{p}>0.05)$ in the SSD group and was significant in the AHL group only at $1 \mathrm{kHz}(\mathrm{p}=0.023)$.

\section{WRS maximum (max \% of recognition at $\mathrm{n} \mathrm{dBHL}$ ) (Figure 4)}

In SSD, the mean $\%$ of WRS maximum was $100 \%$ both in the unaided and BCI-aided situation for mean $\mathrm{dB}$ values of $53.6 \mathrm{~dB}$ (unaided) and 51.4 (BCI-aided). In AHL, the mean \% of WRS maximum was $88 \%$ (unaided) and $98 \%$ (BCI-aided) for mean $\mathrm{dB}$ values of $72 \mathrm{~dB}$ (unaided) and $68 \mathrm{~dB}$ (BCI-aided). When comparing these values, a significant improvement was only observed in the AHL group $(\mathrm{p}=0.028)$. 


\section{Free-Field Speech Audiometry in Quiet (WRS at 65 dB HL)}

In the SSD group, the mean value was $97.3 \%$ in the unaided condition and $99.1 \%$ in the BCIaided condition. In the AHL group, the mean value was $66 \%$ in the unaided condition and $84 \%$ in the BCI-aided condition. The mean gain with BCI was $1.8 \%$ in the SSD group and $18 \%$ in the AHL group, with a difference not statistically significant $(\mathrm{p}>0.05)$.

\section{Speech Reception Threshold (SRT)}

In the SSD group, the mean SRT was $38.4 \mathrm{~dB}$ HL under the unaided condition and $35.7 \mathrm{~dB}$ HL under the BCI-aided condition, with an overall mean gain of $2.7 \mathrm{~dB}$ HL under the latter condition. In the AHL group, the mean unaided SRT was at 59.4 dB HL and the BCI-aided one at $55.8 \mathrm{~dB} \mathrm{HL}$, with an improvement of $3.6 \mathrm{~dB}$ HL. None of these results were significant $(\mathrm{p}>$ $0.05)$.

\section{Free-Field Speech Audiometry in Noise}

The individual and mean maximum WRS in noise $(\mathrm{S} / \mathrm{N}+10)$ in the unaided and BCI-aided conditions are shown in Table IV and Figure 5. In the SSD group, the maximum percentage of intelligibility shifted from $82.7 \%$ (unaided) to $85.5 \%$ (BCI-aided), with a $2.7 \%$ gain. In the AHL group, the maximum percentage of intelligibility shifted from $68 \%$ (unaided) to $79 \%$ (BCI-aided), with an $11 \%$ gain. These values were not statistically significant $(p=0.308)$.

\section{General Glasgow Benefit Inventory}

The mean total score was 10 for the SSD group and 13.8 for the AHL group. In each investigated domain, the mean values were 5.1 (general health), 42.2 (social health) and 3 (physical health) in the SSD group and 11.7 (general health), 33.2 (social health), and 10 (physical health) in the AHL group (Fig. 6). The statistical comparison of the two groups was not significant $(p=0.408)$. 


\section{Visual Analogue Scale}

After three months of BCI use, the mean scores for sound quality were 6.64 in SSD and 7.4 in AHL (Fig. 7), with a significant difference $(\mathrm{p}=0.043)$. The mean scores for life quality were 5.1 in SSD and 6 in AHL, with a non significant difference $(\mathrm{p}=0.076)$.

\section{Entific Medical System QoL}

The mean score was 96.5 in SSD and 98 in AHL. The difference was not statistically significant $(\mathrm{p}>0.05)$ 


\section{DISCUSSION}

BCI are considered a beneficial alternative to conventional CROS hearing aids for rehabilitation of SSD, being able to restore pseudo-stereophonic hearing by artificially recovering the head-shadow effect in presence of a normal or nearly-normal contralateral hearing. Conversely, no agreement exists on the efficacy of applying a BCI when the contralateral hearing is impaired and displays various degrees of functional loss (AHL). While Wazen et al. [6] reported on positive pre-operative tests in subjects with contralateral mild-tomoderate hearing loss (21-54 dB HL), Schwartz and Kobylk [7] remarked that, under these audiological conditions, BCI do not compensate, especially in noisy environments and when noise is presented to the implanted side. However, at the same time, the same authors reported that the better overall sound perception provided by BCI could favourably influence the patient's quality of life [7]. In this regard, much importance has recently been given to the different tests that can evaluate patient satisfaction after auditory implantation in both SSD and AHL cases. When reviewing the literature, it is found that BCI patients prefer to wear their device continuously due to the overall subjective benefits rather than the merely audiological one, with a QoL improvement after few months of BCI use $[13,16]$.

The present study aimed to shed light on the role played by a BCI when the better ear presents with an abnormal hearing function (AHL). For this purpose, this retrospective evaluation has considered two separate groups with similar profound hearing loss in one ear and a different auditory condition in the contralateral ear: normal or near-normal (SSD), or impaired hearing function (AHL).

After BCI implantation, while a $5 \mathrm{~dB}$ gain as free-field PTA was measured in the AHL group, no change was shown in the SSD group due to the well-known ceiling effect related to the normal contralateral hearing. Interestingly, when considering the single frequency gain, the AHL group showed a specific gain at 1 and $4 \mathrm{kHz}$, so that the improvement occurred over a 
frequency range that is critical for speech recognition. Speech audiometry in quiet with BCI showed an improvement in the percentage of intelligibility between $10 \%$ and $18 \%$ in AHL cases, and less than $10 \%$ in the SSD cases, statistically significant in favour of the AHL condition $(\mathrm{p}<0.05)$. This finding supports the hypothesis that, in AHL cases, BCI could induce a contralateral stimulation that is not observed in SSD cases in which the loudness gain is obviously impeded by the presence of the contralateral normal hearing. One may therefore assume that this, although minimal high frequency gain could contribute to the improvement of speech perception in noise, as shown by the better speech in noise test results in the AHL group. The intelligibility gain found only in the AHL group is confirming that the contralateral stimulation with a phase difference, induced by signals to the implanted ear and noise in the other ear, improved speech perception in noise when hearing loss is also present in the nonimplanted ear. The statistical comparison of the groups, however, was not significant also due to the low number of reported cases.

Some further interesting points that are also worth discussing. First, PTA does not seem to be test-specific for verifying the advantages of BCI in SSD, but it is useful in AHL since it may verify the effects of contralateral stimulation and selective frequency improvement. Sound localisation tests have not been used because of the estimated prevalence of the implanted side [11]. Speech audiometry in quiet was shown to be valid only for AHL cases, because the results in SSD patients may be biased by the ceiling effect. Speech audiometry in noise was also shown to be useful only in AHL cases, but since the target is to improve speech perception in difficult listening situations, and in all types of sound and noise direction, a subjective test, such as the Abbreviated Profile of Hearing Aid Benefit, could be considered much more explicative in this regard [18]. The subjective test results were in agreement with what has been previously published [7], with significantly better scores in the AHL group than in the SSD group when evaluating life and sound quality (QoS) by VAS questionnaires. Additionally, the GBI results 
were better in the AHL group than in the SSD group, especially in the general and physicalhealth domains and showed that BCI surgery was not affecting recipients' QoL, in agreement with other published evaluations $[18,19]$. In addition, our study has shown that AHL subjects reported better satisfaction than SSD subjects, although without statistical significance. From the present experience, it seems likely that the conventional battery of audiological tests assessment may not be appropriate for SSD to provide evidence of potential advantages after $\mathrm{BCI}$, although it is useful in cases of AHL, as an effect of contralateral stimulation when contralateral hearing function is impaired and shows selective frequency improvement $(1 \mathrm{kHz})$. It is possible to conclude that BCI did not improve auditory or speech recognition in the SSD group but did, although minimally, improve these factors in the AHL group, with a likely improvement when the contralateral ears present with a mild-to-moderate hearing loss. In both SSD and AHL groups, there was an improvement in subjective sound perception, and a better overall QoL. The rehabilitation of some auditory features that BCI provide in SSD subjects can be positively extended also to AHL cases, leaving the task of restoring binaural hearing in unilateral deafness to cochlear implantation [20]. 


\section{REFERENCES}

1. Usami S, Kitoha R, Motekia H, et al. Etiology of single-sided deafness and asymmetrical hearing loss. Acta Otolaryngol. 2017; 137: suppl. 565.

2. Vincent C, Arndt S, Firszt JB, et al. Identification and Evaluation of Cochlear Implant Candidates with Asymmetrical Hearing Loss. Audiol Neurootol. 2015; 20 Suppl 1:87-9.

3. Vila PM, Lieu JE. Asymmetric and unilateral hearing loss in children. Cell Tissue Res. 2015; 361(1):271-78.

4. Van de Heyning P, Távora-Vieira D, Mertens G, et al. Towards a Unified Testing Framework for Single-Sided Deafness Studies: A Consensus Paper" Audiol Neurootol. 2016; 21(6): 391-98.

5. Sargent EW, Herrmann B, Hollenbeak CS, et al. The minimum speech test battery in profound unilateral hearing loss. Otol Neurotol 2001; 22(4): 480-86.

6. Wazen JJ, Van Ess MJ, Alameda J, et al. The Baha System in Patients with Single Sided Deafness and Contralateral Hearing Loss. Otolaryngol Head Neck Surg. 2010; 142(4):554-59.

7. Schwartz SR, Kobylk D. Outcomes of Bone Anchored Hearing Aids (BAHA) for Single Sided Deafness in Nontraditional Candidates. Otol Neurotol. 2016; 37(10): 1608-13.

8. Arts RA, George EL, Stokroos RJ, et al. Review: cochlear implants as a treatment of tinnitus in single-sided deafness. Curr Opin Otolaryngol Head Neck Surg. 2012;20(5):398-403.

9. Vanecloo FM, Ruzza I, Hanson JN, et al. The monoaural psuedo-stereophonic hearing aid (BAHA) in unilateral total deafness: a study of 29 patients. Rev Laryngol Otol Rhinol (Bord) 2001; 122: 343-50.

10. Niparko JK, Cox KM, Lustig LR. Comparing bone anchored hearing aid implantable hearing device with contralateral routing of offside signal amplification in the rehabilitation of unilateral deafness. Otol Neurotol 2003; 24 (1): 73-8.

11. Lin LM, Bowditch S, Anderson MJ , et al. Amplification in the rehabilitation of unilateral deafness: speech in noise and directional hearing effects with bone-anchored hearing and contralateral routing of signal amplification. Otol Neurotol. 2006; 27(2):17282. 
12. Monini S, Musy I, Filippi C, et al. Bone Conductive Implants in Single- Sided Deafness. Acta Otolaryngol. 2015; 135(4): 381-88.

13. Kim G, Mi JH, Lee SH, et al. Efficacy of Bone-Anchored Hearing Aids in SingleSided Deafness: A Systematic Review. Otol Neurotol. 2017; 38(4):473-83.

14. Monini S, Filippi C, Atturo F, et al. Individualised headband simulation test for predicting outcome after percutaneous bone conductive implantation. Acta Otorhinolaryngol Ital. 2015; 35(4): 258-64.

15. Robinson K, Gatehouse S, Browning GG. Measuring patient benefit from otorhinolaryngological surgery and therapy. Ann Otol Rhinol Laryngol. 1996;105:415-22.

16. Dutt SN, McDermott AL, Jelbert A, et al. Day to day use and service-related issues with the bone-anchored hearing aid: the Entific Medical Systems questionnaire. 2002; J Laryngol Otol Suppl, 28:20-8.

17. Carlson RV, Boyd KM, Webb DJ. The revision of the Declaration of Helsinki: past, present and future. Br J Clin Pharmacol. 2004; 57(6):695-713.

18. Monini S, Bianchi A, Talamonti R, et al. Patient satisfaction after auditory implant surgery: ten-year experience from a single implanting unit center. Acta Otolaryngol. 2017; 137(4): 389-97.

19. Barbara M, Biagini M, Lazzarino AI, et al. Hearing and quality of life in a south European BAHA population. Acta Oto-Laryngologica, 2010; 130 (9):1040-47.

20. Arndt S, Laszig R, Aschendorff A, et al. Cochlear implant treatment of patients with single-sided deafness or asymmetric hearing Loss, HNO, 2017; 65: 98-110. 


\section{LEGENDS}

Figure 1: Mean bone- and air-conduction thresholds of the non-implanted ear in Single-SidedDeafness (SSD) and Asymmetric-Hearing-Loss (AHL) subjects.

Figure 2: Mean Free-field Pure Tone Audiometry thresholds in Single-Sided-Deafness (SSD) and Asymmetric Hearing-Loss (AHL) groups under unaided and aided situations. BCI: Bone Conductive Implant.

Figure 3: Mean gains at each frequency with bone conductive implants (Pure Tone Average 250-4000 Hz) in Single-Sided-Deafness and Asymmetric-Hearing-Loss groups. The gain is significant only at $1000 \mathrm{~Hz}(\mathrm{p}=0.023)$.

Figure 4: Values of maximum Word Recognition Score gain and corresponding dB at speech audiometry in quiet. The greater gain in speech audiometry in quiet of the AHL group in respect to the SSD one, is significant for percent of intelligibility ( $p=0.028)$.

Figure 5: Comparison of percentage of intelligibility (at signal/noise ratio +10 ) in SingleSided-Deafness (SSD) and Asymmetric-Hearing-Loss (AHL) groups. No statistical significance is found within or between the groups ( $p=0.308)$.

Figure 6: General Glasgow Benefit Inventory scores in Single-Sided-Deafness (SSD) and Asymmetric Hearing Loss (AHL) groups. Better total scores are found in the AsymmetricHearing-Loss group; the difference is not statistically significant $(\mathrm{p}>0.05)$.

Figure 7: Visual Analogue Scale scores to assess subjective quality of life (QoL) and sound (QoS) in the Asymmetric-Hearing-Loss and Single-Sided-Deafness groups. Note that the comparison between two groups is statistically significant for QoS $(\mathrm{p}=0.043)$, but not for QoL scores $(p=0.076)$.

Tab. I: Audiological criteria for definition of Single-Sided Deafness (SSD) and Asymmetric Hearing Loss (AHL) [from Vincent at al., 2015]. 
Tab. II: Individual and mean Pure Tone average (PTA) values before and after BCI Implant, with gain in the SSD and AHL groups.

Tab. III: Individual and mean values of maximum percentage of Word Recognition Score (WRS) and corresponding dB HL, at speech audiometry in quiet, in the Single Sided Deafness (SSD) and Asymmetric Hearing Loss (AHL) groups.

Tab. IV: Percentage of intelligibility in free-field speech audiometry, under the unaided and the BCI-aided condition, for Single-Sided Deafness (SSD) and Asymmetric Hearing Loss (AHL). 
Figure 2: Mean Free-field Pure Tone Audiometry thresholds in Single-Sided-Deafness (SSD) and Asymmetric Hearing-Loss (AHL) groups under unaided and aided situations. BCI: Bone Conductive Implant.

$209 \times 297 \mathrm{~mm}(300 \times 300 \mathrm{DPI})$ 
Figure 3: Mean gains at each frequency with bone conductive implants (Pure Tone Average 250-4000 $\mathrm{Hz}$ ) in Single-Sided-Deafness and Asymmetric-Hearing-Loss groups. The gain is significant only at $1000 \mathrm{~Hz}(p=$ $0.023)$.

$209 \times 297 \mathrm{~mm}(300 \times 300$ DPI $)$ 

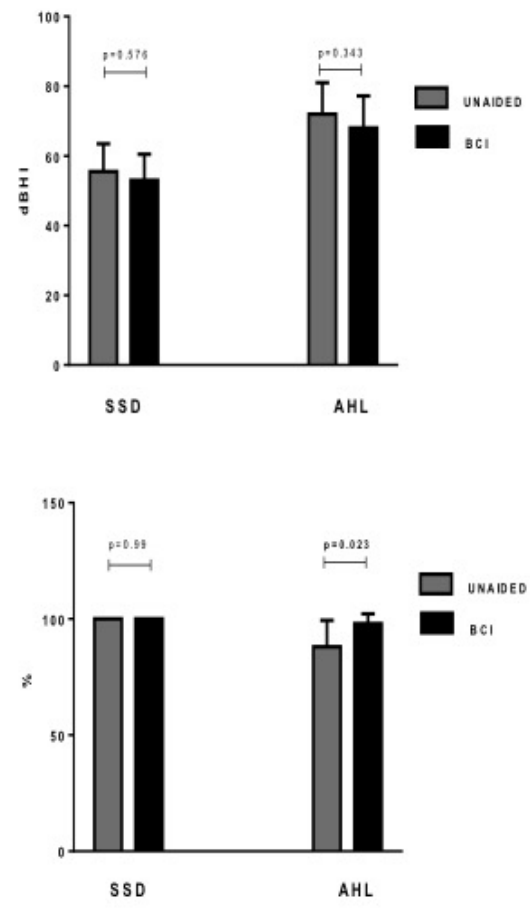

Figure 4: Values of maximum Word Recognition Score gain and corresponding dB at speech audiometry in quiet. The greater gain in speech audiometry in quiet of the AHL group in respect to the SSD one, is significant for percent of intelligibility $(p=0.028)$.

$254 \times 190 \mathrm{~mm}(72 \times 72 \mathrm{DPI})$ 




S S D

\section{AHL}

Figure 5: Comparison of percentage of intelligibility (at signal/noise ratio +10 ) in Single-Sided-Deafness (SSD) and Asymmetric-Hearing-Loss (AHL) groups. No statistical significance is found within or between the groups $(p=0.308)$.

$195 \times 186 \mathrm{~mm}(72 \times 72 \mathrm{DPI})$ 
Figure 6: General Glasgow Benefit Inventory scores in Single-Sided-Deafness (SSD) and Asymmetric Hearing Loss $(A H L)$ groups. Better total scores are found in the Asymmetric-Hearing-Loss group; the difference is not statistically significant $(p>0.05)$.

\section{$273 \times 178 \mathrm{~mm}(72 \times 72 \mathrm{DPI})$}






Figure 7: Visual Analogue Scale scores to assess subjective quality of life (QoL) and sound (QoS) in the Asymmetric-Hearing-Loss and Single-Sided-Deafness groups. Note that the comparison between two groups is statistically significant for QoS $(p=0.043)$, but not for QoL scores $(p=0.076)$.

$205 \times 151 \mathrm{~mm}(72 \times 72 \mathrm{DPI})$ 


\begin{tabular}{|l|l|l|}
\hline \multirow{4}{*}{ SSD } & Poorer ear & $\begin{array}{l}\text { Severe-to-profound } \\
\text { hearing loss }\end{array}$ \\
\cline { 2 - 3 } & Better ear & $<30 \mathrm{~dB}$ HL up to $4 \mathrm{kHz}$ \\
\cline { 2 - 3 } & $\begin{array}{l}\text { Interaural threshold } \\
\text { difference }\end{array}$ & $\geq 40 \mathrm{~dB} \mathrm{HL}$ \\
\hline \multirow{4}{*}{ AHL } & Poorer ear & $\begin{array}{l}\text { Severe-to-profound } \\
\text { hearing loss }\end{array}$ \\
\cline { 2 - 3 } & Better ear & $>30 \mathrm{~dB}$ HL \\
& Interaural threshold & $60 \mathrm{~dB}$ HL up to $4 \mathrm{kHz}$ \\
\cline { 2 - 3 } & difference & $\geq 15 \mathrm{~dB} \mathrm{HL}$ \\
\hline
\end{tabular}

Table I 
FREE FIELD PURE TONE AUDIOMETRY

\begin{tabular}{|c|c|c|c|}
\hline PATIENT & $\begin{array}{c}\text { UNAIDED } \\
\text { (dBHL) }\end{array}$ & $\begin{array}{c}\text { BCI- } \\
\text { AIDED } \\
\text { (dBHL) }\end{array}$ & $\begin{array}{l}\text { PTA GAIN } \\
\text { (dBHL) }\end{array}$ \\
\hline \multicolumn{4}{|c|}{ SSD } \\
\hline 1 & 35 & 35 & 0 \\
\hline 2 & 52 & 51 & 1 \\
\hline 3 & 50 & 46 & 4 \\
\hline 4 & 17 & 17 & 0 \\
\hline 5 & 50 & 48 & 2 \\
\hline 6 & 31 & 29 & 2 \\
\hline 7 & 41 & 41 & 0 \\
\hline 8 & 59 & 55 & 4 \\
\hline 9 & 40 & 30 & 10 \\
\hline 10 & 20 & 15 & 5 \\
\hline 11 & 17 & 17 & 0 \\
\hline MEAN & 37,5 & 34,9 & 2,5 \\
\hline SD & 8 & & 14,35149358 \\
\hline \multicolumn{4}{|c|}{ AHL } \\
\hline 12 & 67 & 58 & 9 \\
\hline 13 & 67 & 65 & 2 \\
\hline 14 & 58 & 57 & 1 \\
\hline 15 & 43 & 44 & -1 \\
\hline 16 & 72 & 73 & -1 \\
\hline 17 & 63 & 56 & 7 \\
\hline 18 & 60 & 42,6 & 17,4 \\
\hline 19 & 66 & 57 & 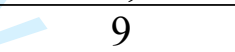 \\
\hline 20 & 68 & 61 & 7 \\
\hline 21 & 40 & 39 & 1 \\
\hline MEAN & 60,4 & 55,3 & 5,1 \\
\hline SD & & & 10,71786998 \\
\hline
\end{tabular}

Table II 


\begin{tabular}{|c|c|c|c|c|c|c|}
\hline PTS & $\begin{array}{c}\text { WRS } \\
\text { MAX } \\
\text { (dBHL) } \\
\text { UNAIDED }\end{array}$ & $\begin{array}{c}\text { WRS } \\
\text { MAX (\%) } \\
\text { UNAIDED }\end{array}$ & $\begin{array}{c}\text { WRS } \\
\text { MAX } \\
\text { (dBHL) } \\
\text { BCI- } \\
\text { AIDED } \\
\end{array}$ & $\begin{array}{c}\text { WRS } \\
\text { MAX } \\
\text { (\%) } \\
\text { BCI- } \\
\text { AIDED } \\
\end{array}$ & $\begin{array}{c}\text { MAX WRS } \\
\text { GAIN } \\
\text { (dBHL) }\end{array}$ & $\begin{array}{c}\text { MAX WRS } \\
\text { GAIN (\%) }\end{array}$ \\
\hline \multicolumn{7}{|c|}{ SSD } \\
\hline 1 & 60 & 100 & 55 & 100 & 5 & 0 \\
\hline 2 & 50 & 100 & 55 & 100 & -5 & 0 \\
\hline 3 & 55 & 100 & 50 & 100 & 5 & 0 \\
\hline 4 & 50 & 100 & 40 & 100 & 10 & 0 \\
\hline 5 & 75 & 100 & 70 & 100 & 5 & 0 \\
\hline 6 & 55 & 100 & 55 & 100 & 0 & 0 \\
\hline 7 & 50 & 100 & 50 & 100 & 0 & 0 \\
\hline 8 & 50 & 100 & 50 & 100 & 0 & 0 \\
\hline 9 & 60 & 100 & 55 & 100 & 5 & 0 \\
\hline 10 & 50 & 100 & 50 & 100 & 0 & 0 \\
\hline 11 & 35 & 100 & 35 & 100 & 0 & 0 \\
\hline MEDIA & 53,6 & 100 & 51,4 & 100 & 2,3 & 0 \\
\hline SD & & 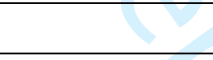 & & & 9,22 & 0 \\
\hline \multicolumn{7}{|c|}{ AHL } \\
\hline 12 & 70 & 100 & 55 & 100 & 15 & 0 \\
\hline 13 & 80 & 80 & 80 & 90 & 0 & 10 \\
\hline 14 & 80 & 100 & 65 & 100 & 15 & 0 \\
\hline 15 & 70 & 80 & 65 & 100 & 5 & 20 \\
\hline 16 & 80 & 70 & 80 & 90 & 0 & 20 \\
\hline 17 & 60 & 90 & 80 & 100 & -20 & 10 \\
\hline 18 & 80 & 80 & 70 & 100 & 10 & 20 \\
\hline 19 & 80 & 80 & 65 & 100 & 15 & 20 \\
\hline 20 & 60 & 100 & 60 & 100 & 0 & 0 \\
\hline 21 & 60 & 100 & 60 & 100 & 0 & 0 \\
\hline MEDIA & 72 & 88 & 68 & 98 & 4 & 10 \\
\hline SD & & & & & 9,176629355 & 9,787209699 \\
\hline
\end{tabular}

Table III 
FREE FIELD SPEECH AUDIOMETRY IN NOISE

\begin{tabular}{|c|c|c|c|}
\hline PTS & $\begin{array}{c}\text { \% MAX } \\
\text { INTELLIGIBILITY } \\
\text { UNAIDED }\end{array}$ & $\begin{array}{c}\text { \% MAX } \\
\text { INTELLIGIBILITY } \\
\text { BCI-AIDED }\end{array}$ & GAIN \\
\hline \multicolumn{4}{|c|}{ SSD } \\
\hline 1 & 80 & 90 & 10 \\
\hline 2 & 100 & 100 & 0 \\
\hline 3 & 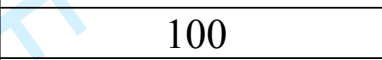 & 70 & -30 \\
\hline 4 & 2 & 80 & 0 \\
\hline 5 & $\begin{array}{r}70 \\
\end{array}$ & 80 & 10 \\
\hline 6 & $P$ & 80 & 0 \\
\hline 7 & 100 & 90 & -10 \\
\hline 8 & 60 & 100 & 40 \\
\hline 9 & 80 & 80 & 0 \\
\hline 10 & 90 & 90 & 0 \\
\hline 11 & 70 & 80 & 10 \\
\hline MEDIA & 82,7 & 85,5 & 2,7 \\
\hline SD & 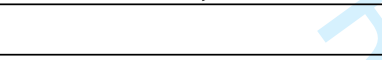 & D & 11,40555041 \\
\hline \multicolumn{4}{|c|}{ AHL } \\
\hline 12 & 100 & 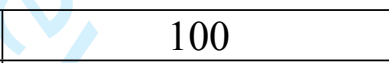 & 0 \\
\hline 13 & 50 & 50 & 0 \\
\hline 14 & 90 & 100 & 10 \\
\hline 15 & 80 & 90 & 10 \\
\hline 16 & 70 & 80 & 10 \\
\hline 17 & 70 & 80 & 10 \\
\hline 18 & 70 & 70 & 0 \\
\hline 19 & 20 & 80 & 60 \\
\hline 20 & 50 & 8 & -10 \\
\hline 21 & 80 & 100 & 20 \\
\hline MEDIA & 68 & 79 & 11 \\
\hline SD & & 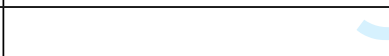 & 22,07046133 \\
\hline
\end{tabular}

Table IV 